\title{
Basics of Managed Care Claims Processing: From Claims Payment to Outcomes Management
}

Kenneth W. Schafermeyer

OBJECTIVE:

Describe the evolution of prescription claims processing from claims payment systems designed in the 1970 s to interactive point-of-service (POS) systems that enable pharmacists to conduct prospective drug-use review; and discuss current programs that link prescription databases with medical, hospital, laboratory, and other health care records; and explore the benefits and limitations of electronic point-ofservice systems for providers, payers, and patients.

\section{DATA SOURCES:}

Not applicable.

\section{DATA EXTRACTION:}

Not applicable.

\begin{abstract}
DATA SYNTHESIS:
Claims-processing systems have developed quickly from the completion of manual forms in the 1970s, to standardization of forms using the National Drug Code and the National Pharmacy Identification Code; to the real-time point-of-service (POS) systems of the 1990s that provide management functions. POS systems benefit the pharmacy, patient, and pharmacy benefit management company. The capability of performing prospective drug-use review is one of the most important advantages of POS systems. On-line systems also form the basis for measuring the outcomes produced by a particular pharmacy and for conducting outcomes research studies.
\end{abstract}

\section{CONCLUSION:}

Claims databases will be used more for research, patient case management, and evaluating the performance of pharmacists and other health care providers.

\section{KEY WORDS:}

Third-party prescription programs, National Council for Prescription Drug Programs, Universal Claim Form, National Association of Boards of Pharmacy, National Pharmacy Identification Code, Real-time transactions, Point-of-service systems, Prospective drug-use review, Managed care organizations, Pharmacy benefit managers, Drug product selection codes, Dispense as written codes, Network switches.

J Managed Care Pharm 1995: 1: 200-205.
Claims processing has evolved over the past quarter of a century from tedious manually completed forms to today's on-line, real-time claims adjudication systems. Through the capture of immediate data about drug-use in a covered population, pharmacists and plan administrators find themselves witnessing the emergence of a new way of processing, tracking, and paying for prescription drugs.

This article describes the evolution of prescription claims processing from the 1970 s to the present; discusses programs that currently link prescription databases with other health care records; and explores the benefits and limitations of electronic point-of-service systems.

\section{EVOLUTION OF CLAIMS PROCESSING}

In the early days of third-party prescription programs, claims-processing systems were designed simply to pay claims. Pharmacists completed claims by hand, an arduous task further complicated before 1976 by each plan using its own form. The result was that pharmacists had to stock copies of multiple claim forms that requested different information and had to learn the requirements and idiosyncrasies of each plan. Claim forms required that 80 or more digits be filled in to properly record: pharmacy identification numbers, plan identification numbers, patient identification numbers, drug code numbers, and information such as quantity, days supply, and refills authorized. Additionally, each plan had its own system of identification numbers and its own drug coding system. Needless to say, completing claim forms consumed a considerable amount of time and was prone to error. Even if pharmacists completed claim forms correctly, the plan's keypunchers had to correctly transcribe the information from the paper claims to a computer-readable format. Unless the plan used a doubleentry verification system, many errors could occur at this

\section{Author}

KENNETH W. SCHAFERMEYER, R.PH., PH.D., is Associate Professor of Pharmacy Administration, St. Louis College of Pharmacy, St. Louis, MO.

Portions of this article were adapted from Schafermeyer KW, McCann SD. Point-of-sale processing of medicaid prescription claims. Missouri Pharmacist 1992(Nov); 66(11): 14-7. The author expresses his appreciation to Eric H. Hobson, PhD for assistance with the manuscript.

Copyright 0 1995, Academy of Managed Care Pharmacy, Inc. All rights reserved. 
point as well. The result was suboptimal: prescription claims processing was confusing, time consuming, frustrating, expensive, and inefficient.

In 1976, the National Council for Prescription Drug Programs (NCPDP) was formed to standardize third-party claim forms and ease the burden of third-party claims submissions. The result was the Universal Claim Form, eventually adopted by most third-party payers. NCPDP's roots go back to the "Drug Ad Hoc Committee" formed in 1972 to assist the Food and Drug Administration in developing and promoting the use of a numeric national drug code (NDC). Later, NCPDP and the National Association of Boards of Pharmacy (NABP) standardized a National Pharmacy Identification Code (NPIC), which provides a seven-digit identifier for each pharmacy in the U.S. participating in a third-party prescription program.

Soon, high-volume pharmacies found it more efficient to have computers print pin-fed claim forms in large batches. Never ones to miss a market opportunity, computer vendors soon offered tape billing services, decreasing the amount of paper handling required for claims processing. Now, magnetic tape media are disappearing as most plans and pharmacies are adopting real-time transaction processing-completing the adjudication of a claim while the patient is in the pharmacy. These modem-to-modem transmission systems, commonly known as "point-of-service" (POS) systems, provide five distinct management functions: (1) eligibility verification; (2) prior authorization for certain drugs; (3) claims submission; (4) claims adjudication; and (5) prospective drug-use review (ProDUR). Complexity, as well as potential benefits, increases with the addition of each new function.

One function that has not been automated is obtaining patients' signatures to verify that prescriptions have been received. While signatures were once required on each paper claim form, most managed care programs now require patients to sign signature logs containing prescription numbers and dates received. Some Medicaid and managed care patients sign an additional section of the log, documenting that pharmacists have offered to counsel them about proper medication use.

Essentially an enhancement of programs originally designed to verify credit card eligibility, early pharmacy POS technology is limited to a relatively simple eligibility verification function: information was entered by passing a magnetic card through a "swipe card" machine or by keying information into a "black box" or computer terminal linked to a vendor's central computer by a telephone line. Eligibility verification benefits both pharmacies and the third parties by reducing the time and cost of dealing with rejected claims.

However, expanding this basic eligibility verification system to include other POS claims management functions is a much more complex undertaking. For instance, adding claims submission and adjudication capabilities requires constant maintenance of NDC numbers, product price updates, changes in program specifications, and myriad other on-going tasks.
The rapid increase in the number of managed care organizations (MCOs) and pharmacy benefit managers (PBMs) — and the increased percentage of prescriptions paid by these plans-necessitated the development of a standard format for the submission of electronic claims. NCPDP addressed this need by releasing "Telecommunication Standard Format Version 3.2" in February 1992. This format, which most MCOs and PBMs have adopted, was designed to meet the Pro-DUR requirements of OBRA ' 90 and to enable the documentation and payment of additional cognitive services such as patient monitoring and intervention.

However, many payers have not adopted all NCPDP coding standards. For example, recent interviews of $100 \mathrm{MCO}$ and PBMs found that many have not adopted the drug product selection codes, formerly known as the dispense as written (DAW) codes, to the extent expected. This omission has led some observers to speculate that some MCOs and PBMs may have chosen not to recognize some DAW codes to discourage the use of brand name drugs and, therefore, to save moneyeven though this may create additional expense and inconvenience for patients and pharmacies. ${ }^{1}$ Part of this problem could be attributed to computer vendors who have not provided software updates in a timely manner, and pharmacists who have not installed these updates when provided.

Even with a standard electronic processing format, communicating on-line with a host of different third-party processors creates a number of headaches for pharmacies.

Transmitting real-time claims directly to payers sometimes requires pharmacies to lease individual telephone lines for each processor. Processors receiving real-time claims directly from pharmacies have to: (1) establish and support a communications network; (2) develop support desks to deal directly with pharmacy providers; and (3) write communications protocols that can be used by the individual pharmacies. As might be expected, the solution to this confusion has spawned a new industry, network switches, that offers a single source for sending claims to the proper destinations and returning information and payment back to the pharmacy. While network switches have been used for years in credit-card authorizations, this technology has been applied to prescription claims processing only within the past few years. Presently, about $70 \%$ of real-time claims are routed through a switch. ${ }^{2}$

National Data Corporation (NDC) was the first company to provide switching services, soon followed by Envoy and General Computer Corporation (GCC). NDC and GCC operate as both switches and claims processors; Envoy concentrates solely on network switching.

\section{BENEFITS OF POS SYSTEMS}

POS claims processing benefits pharmacy providers, payers, and patients. By linking plans to pharmacies at the time that a prescription is dispensed, POS systems help pharmacists:

$\boldsymbol{\nabla}$ Verify that the patient is eligible for coverage 
$\boldsymbol{\nabla}$ Determine if the prescribed medication is covered

$\nabla$ Determine if there is a maximum allowable cost (MAC)

for multi-source medications

$\nabla$ Determine the maximum amount that may be dispensed

$\boldsymbol{\nabla}$ Monitor refills to assure they are not dispensed too early

$\boldsymbol{\nabla}$ Check for possible drug-drug interactions

$\boldsymbol{\nabla}$ Confirm the amount of co-payment and the net amount to be paid by the plan

$\boldsymbol{\nabla}$ Submit the claim for payment

Pharmacies benefit from POS systems because improved accuracy results in fewer claims rejections. Additionally, claims-processing time is reduced, accelerating payment and improving cash flow, resulting in lower overhead costs for pharmacies dispensing third-party prescriptions. According to Norwood and Lipson, ${ }^{3}$ manually filing third-party claim forms takes about five minutes; on the other hand, electronic data processing reduces this to about one minute. In some pharmacies, this time savings translates to lower personnel costs; in others, it means more time for other tasks.

Confirming recipient eligibility and checking for completeness and accuracy of claims at the time of submittal not only saves time from correcting errors (and the concurrent frustration) but also translates directly into substantial cost savings. As explained by a PAID Prescriptions executive, "If a pharmacy is experiencing a rejection rate of even as low as $2 \%$, this is 40 cents on an average claim of $\$ 20$, compared with the 12 cent transaction charge. The pharmacist still comes out ahead. ${ }^{4}$ This statement was made in 1988; today's higher average claim costs make the argument even more persuasive.

The potential savings that a pharmacy may realize through electronic claims processing depends on a multitude of factors, including the pharmacy's prescription volume, its percentage of managed care prescriptions, and the ratio of pharmacists to supportive personnel in the prescription department. ${ }^{5}$ However, the amount of actual savings that may accrue to pharmacies has not yet been quantified in any published research

Other benefits that accrue to the pharmacy are reduced paper volume, accelerated payment, improved cash flow, faster responses to prior authorization requests, and improved ability to perform Pro-DUR (discussed in more depth later in this article). POS claims processing has great potential to enhance the pharmacist's ability to participate in disease state management and patient outcomes management. Standardized coding systems will enable pharmacists to automate record keeping for patient monitoring and interventions. This capability, together with real-time links to medical, hospital, and laboratory databases, will offer pharmacists increased opportunities to demonstrate, and possibly be reimbursed for, improvement of patient outcomes.

Obviously, MCOs and PBMs also benefit from electronic processing of third-party claims-otherwise, they would not be developing the systems. POS systems reduce the number of data-entry operators because pharmacists perform the data entry. Accepting clean data that has already been screened eliminates the inconvenience and cost of claims suspensions and rejects in secondary to data-entry errors. Contrary to what some pharmacists may believe, PBMs benefit from reducing the number of claims rejections and speeding up claims payments-they usually get paid for claims only after adjudication. Therefore, claims suspensions and denials create additional work for PBMs, not additional income.

PBMs and MCOs also benefit by making use of the valuable marketing information contained in claims databases. Pharmaceutical manufacturers are willing to pay for information that gives detailed information about the drug prescribing and usage patterns so that they can:

$\boldsymbol{\nabla}$ Determine the market share of a product and its competitors

$\boldsymbol{\nabla}$ Evaluate the effectiveness of marketing efforts- especially for sales representatives

$\boldsymbol{\nabla}$ Identify prescribers who have (or have not) responded to marketing efforts

$\boldsymbol{\nabla}$ Determine how a medication is actually used in real settings-diagnoses, quantities prescribed, numbers of refills, concurrent therapy, and length of therapy

$\boldsymbol{\nabla}$ Compare usage levels to expectations-especially in those cases in which formularies alter normal usage patterns

A POS system can reduce costs for both pharmacies and payers by streamlining the processing of prior authorization requests and allowing pharmacists access to patient case-management information that may not otherwise be available.

Patients also benefit from the simplified dispensing and verification processes offered by POS systems. On-line verification of coverage for particular drugs avoids unnecessary prescription denials and eliminates the need for billing patients later for denied claims. With prior authorization requests streamlined from a few days to just a few minutes or less, patients can sometimes receive their medications faster.

Perhaps the greatest benefit currently accruing to all parties, and especially to patients, is the improved ability to perform Pro-DUR. ${ }^{6}$ The consolidation of information into a centralized data pool enhances pharmacists' abilities to review drug-use patterns, monitor refill compliance, and screen for drug-drug interactions and other incompatibilities. Extreme care, of course, must be taken to protect the patients' rights to privacy - most plans have extensive policies and procedures protecting patient confidentiality and limiting access to patient records.

With all of the advantages of POS processing, why are some claims still processed manually? According to the Workgroup for Electronic Data Interchange-a task force appointed by the Department of Health and Human Services-the Medicare program faces several impediments in complete conversion to electronic claims processing. These include the cap- 
ital investment and training costs required to convert to an electronic system, fear of current technology bécoming obsolete, resistance to change, state laws prohibiting the exchange of claims information, and the lack of incentives to convert to an electronic system. ${ }^{7}$

Some pharmacies are not eager to convert to POS processing for all plans because of the implementation costs incurred when switching to electronic claims processing. These initial costs include: pharmacy computer software, lease or purchase of special equipment, installation costs, software support costs, hardware upgrades, telephone cost, and the cost of training personnel to use the system. On-going costs, ranging from approximately 8 to 25 cents, are incurred with each claim transmitted. In some areas, pharmacies may incur another $8-10$ cents in telephone charges to access the network: 8

\section{PROSPECTIVE DRUG-USE REVIEW}

For more than 15 years, many PBMs have routinely reviewed their prescription claims databases to study drug prescribing, dispensing, and patients' drug-usage patterns to detect problems with inappropriate prescribing, drug misuse, and over- and under-use. Since these reviews are based on prescriptions already dispensed, they are referred to as "retrospective drug-use review." The focus of these programs was educational, based on the hope that alerting physicians and pharmacists to potential problems and suggesting ways to improve drug therapy would lead to more effective-and less expensive-drug usage.

The adoption of POS claims processing allows pharmacists to detect and prevent problems before a prescription is dispensed. Pro-DUR programs result in an alert message being sent to the pharmacy's computer whenever problems are detected with the following:

$\nabla$ Dosage out of normal range

$\nabla$ Drug-drug interactions (categorized according to severity)

$\boldsymbol{\nabla}$ Drug-disease contraindications

$\nabla$ Drug-allergy contraindications

$\boldsymbol{\nabla}$ Duplicate therapy

$\nabla$ Side effects

$\nabla$ Refills too early or too late

$\nabla$ Duration of therapy exceeds recommendations

POS systems usually provide pharmacists with pop-up messages on their computer screens. Pharmacists may then take one of two courses of action:

Cancel the prescription Usually, the pharmacist will contact the prescriber, who can cancel the therapy and/or select a different medication.

\section{Override the alert and dispense the prescription}

If the prescriber and/or pharmacist determines that no potential problem exists for the patient in question, the alert may be overridden. In some cases, the pharmacist may not be allowed to.override the alert (i.e., drug-drug interactions that are very serious or life-threatening). An override notification becomes part of the patient's record.

The most common alert message-early refill-is commonly overridden by pharmacists because:

$\boldsymbol{\nabla}$ The physician has told the patient to increase the daily dose

$\nabla$ Patients may come in for other refills and want to avoid making another trip a few days later for the refill in question

vacations

The Pro-DUR process offers the greatest benefit of POS technology for pharmacy providers. Pharmacists counseling patients who patronize more than one pharmacy will no longer be limited to the prescription information from their pharmacy alone. It is expected that the information provided through the Pro-DUR feature of POS systems will help pharmacists enhance professional services.

\section{LINKING PROVIDERS AND INTEGRATING DATA}

As effective as Pro-DUR may be, it does have a few limitations. First, it is often difficult to make appropriate judgments regarding drug therapy without complete information regarding diagnosis, co-morbidities, or needed laboratory data. However, many MCOs have separated the pharmacy plan from the main health care plan by using separate management and fee structures. This separation, known as a "carve-out," can limit the extent to which MCOs can integrate prescription data with data for medical, hospital, and other health care services. Unless medical data are integrated, it is difficult to assess the impact of pharmacy services on overall health care costs.

Many MCOs can integrate various medical claims databases for retrospective reports and reviews; however, complete, integrated databases for use by providers at the time services are provided are not common outside staff model HMOS. One of the first programs to provide these on-line linkages was introduced by PCS in 1994 as "RECAP Plus."

By creating direct electronic linkages with prescribers, as well as pharmacies, payers can incorporate drug-use information with medical data in the physician's office. This should lead to more rational prescribing and prevent situations in which pharmacists must contact physicians to correct problems such as duplicate therapy or unusual patient usage and refill patterns detected at the time of dispensing. Making prescription drug-use information available to physicians can save pharmacists and patients time and frustration. Additional features available to physicians are: formulary status verification, alternative drug therapy suggestions, drug-cost information, patient drug-use histories, and many of the PróDUR alerts previously mentioned.

Pharmacies can benefit through a decrease in the number of interventions needed, allowing electronic refill requests, and assisting in compliance management. Staff model HMOs have always had the advantage of allowing pharmacists greater access to complete patient records; electronic linkages of vari- 
ous providers can allow pharmacists contracting with network and independent practice association HMOs some of the same patient-management opportunities.

\section{MEASURING PHARMACIES' OUTCOMES}

Some managed care organizations find it advantageous to contract with some pharmacies while excluding others. Usually, preferred pharmacies are selected based on factors such as cost, location, size, and volume. Increasingly, there is interest in selecting pharmacies based on their level of service and their potential to produce positive patient outcomes. By comparing pharmacy providers, MCOs can prepare "report cards" showing how pharmacies adhere to preselected performance criteria. Some areas in which MCOs currently compare pharmacies include the following?:

$\boldsymbol{\nabla}$ Acceptance of low reimbursement

$\nabla$ Generic dispensing

$\nabla$ Therapeutic interchange

$\boldsymbol{\nabla}$ 24-hour emergency service

$\nabla$ Desirable location

$\nabla$ Unblemished professional license

$\boldsymbol{\nabla}$ Customer satisfaction

$\nabla$ Appropriate computer software

$\boldsymbol{\nabla}$ Responsiveness to the plan's reports and analyses

$\boldsymbol{\nabla}$ Professional training

$\boldsymbol{\nabla}$ Willingness to undergo audits and inspections

$\nabla$ Professional liability insurance

$\boldsymbol{\nabla}$ Good credit rating

$\nabla$ Satisfactory counseling behavior

$\nabla$ Willingness to accept Medicaid patients

$\nabla$ Formulary compliance

$\boldsymbol{\nabla}$ Clean, modern store

$\boldsymbol{\nabla}$ Competitive usual-and-customary rates

$\nabla$ Depth of stock

Plans vary in the amount of weight given to each of these areas. However, it should be noted that these performance criteria are measures of inputs, not outcomes. Ideally, patient outcomes would be measured in terms of cures, alleviation of symptoms, avoidance of relapse, or decreased use and expense for other health care services such as physician office visits, laboratory tests, hospitalization, and nursing home admissions. Since payers are interested in overall costs, perhaps the best outcome measurement for the MCO's purposes would be total health care costs per patient per year. Of course, these measurements would have to be indexed according to age and gender of each pharmacy's patrons to be comparable.

Obviously, measuring outcomes is a complex task. While measuring outputs and rewarding providers according to performance is the ultimate goal, MCOs find it easier to measure and incite inputs under the reasonable assumption that the desired results can only be obtained if the proper inputs (i.e., structures and processes) are in place; therefore, inputs are often used as surrogate indicators of outcomes. Examples of structure may be a clean modern pharmacy, a private area for patient counseling, and 24-hour emergency services. It seems intuitive (whether it is actually true or not) that a pharmacy could not affect outcomes as well without some location for private patient counseling (a structural input) and enough time to allow counseling as well as written store policies and procedures encouraging patient counseling (procedural inputs).

National Prescription Administrators (NPA), a New Jersey-based PBM, has been classifying pharmacies since 1979 according to their self-reported profiles in areas such as patient counseling, generic substitution, 24-hour emergency services, maintenance dispensing, and other services. Audits are conducted to assure that the pharmacies are classified properly. ${ }^{10}$

In the foreseeable future, it is reasonable to assume that MCOs will select pharmacies according to their expertise in disease state management. A rational assumption is that pharmacists can more effectively improve patient outcomes if they have the proper inputs, such as:

$\boldsymbol{\nabla}$ Education in managing selected disease states, perhaps through recognized certification programs (a structural input)

$\nabla$ Proper pharmacy layout allowing patient counseling in a private atmosphere (a structural input)

$\boldsymbol{\nabla}$ A computer system with on-line capability and the ability to facilitate Pro-DUR (a structural input)

$\boldsymbol{\nabla}$ Adequate time, evidenced by a number of prescriptions per hour per pharmacist, that does not exceed a given volume (a procedural input)

Of course, no claims-processing system alone is enough for credentialing purposes. However, on-line capability would be a basic requirement. Claims databases could verify generic dispensing rates, formulary compliance, therapeutic interchange rates, and cost per patient per year.

\section{MANAGING OUTCOMES}

The term "managed care" implies that payers will define, measure, and assure the quality and cost-effectiveness of health care services. To do this, MCOs need to make even greater use of prescription claims databases. Although prescription claims databases were originally designed for reimbursement purposes, on-line links with medical, hospital, and other health care claims allow them to be adapted for outcomes research. Outcomes studies are no longer restricted to clinical trials-they can also be conducted in real health care settings.

Compared with clinical trials and postmarketing surveillance, claims databases offer several advantages for outcomes research, including the following ${ }^{11}$ :

$\boldsymbol{\nabla}$ Longitudinal studies may be conducted for chronic diseases

$\boldsymbol{\nabla}$ Relatively uncommon diseases may be selected for study

$\boldsymbol{\nabla}$ Defined subgroups that are often overlooked in clinical trials, such as women and children, may be studied

$\boldsymbol{\nabla}$ Reimbursement information may be used for economic studies 
$\nabla$ Patient recall or provider bias is eliminated

$\nabla$ Less time and expense is required than for clinical trials

However, claims databases also have some limitations. First, they are, by nature, incomplete-they do not include information that is irrelevant to insurance reimbursement, such as use of nonformulary drugs, nonprescription medications, and samples. Second, vital diagnostic information is often incomplete or incorrect. Diagnoses do not usually indicate a condition's severity or co-morbidities. When a diagnosis can be determined, it is often difficult to determine the cause. For example, was the patient's $\mathrm{H}_{2}$ antagonist given to treat an existing gastric ulcer or to counteract gastric irritation of a nonsteroidal anti-inflammatory drug? Third, claims databases record drug use but usually not the consequences of that use-such as the degree of efficacy or adverse effects.

When a diagnosis is not coded properly on claim forms, it is sometimes possible to infer a diagnosis from the drug therapy. For example, patients whose diabetes is not controlled by diet alone can be easily detected by their use of insulin or oral hypoglycemic agents.

\section{CONCLUSION}

Despite these limitations, claims databases will be used more and more for research, patient case management, and evaluating the performance of pharmacists and other healthcare providers. POS claims-processing systems and integration of all health care claims are, of course, prerequisites. The possibilities and advantages of electronic claims processing are clearly only beginning to be realized. The future prosperity, and even survival, of managed care programs and participating providers will depend upon the extent to which this technology is used for the benefit of patient care.

\section{$\nabla \quad$ References}

1. Anonymous. Survey indicates misunderstanding and misuse of DAW codes. Council Connection 1995 (May/Jun); 1 (3): 5-7.

2. Anonymous. Network switches: the third-party connection. ComputerTalk Pharm 1992

(Sep/Oct); 12 (5): 25.

3. Norwood GJ, Lipson DP. Computerization in pharmacy: management and professional implications. J Am Pharm Assoc 1977 (Mar); NS17 (3): 177-9.

4. Anonymous. Electronic claims: a revolution in progress. ComputerTalk Pharm 1988 (Sept/Oct); $8(5): 15-6$.

5. Norwood G], Lipson DP. Computerization in pharmacy: management and professional implications. J Am Pharm Assoc 1977 (Mar); NS17 (3): 177.

6. Sturek JK. Point of service computer systems and drug use evaluation: implications for pharmacy practice in ambulatory care. Am J Hosp Pharm 1988; 46 (Suppl): S17-8.

7. Cantrell SA. Electronic processing of medicare claims. Am J Hosp Pharm 1993; 50: 424.
8. Bartone D. Transaction charges: an unfair levy. NARD 1994 (Jun); $116(6): 65$.

9. Marcille JA. Contracting: how will you measure up? Managed Care Pharm Pract 1995 (May/Jun); 116 (6): 65

10. Marcille JA. Contracting: how will you measure up? Managed Care Pharm Prac 1995 (May/Jun); $116(6): 65$.

11. Lewis NJW, Patwell JI, Briesacher BA. The role of insurance claims databases in drug therapy outcomes research. PharmacoEconomics 1993; 4(5): $324-5$ 